\title{
Effects of Changes in Physical Activity with Cognitive Decline in Korean Home-Dwelling Older Adults
}

\author{
Hyunjong Song', Jin-Hwa Park ${ }^{2}$ \\ 'Department of Health Policy and Management, College of Health Sciences, Sangji University, Gangwon-do, Republic of Korea; ${ }^{2}$ College of Nursing, \\ Institute of Nursing Science, Daegu Catholic University, Daegu, Republic of Korea \\ Correspondence: Jin-Hwa Park, Tel +82 53650 4754, Fax +82 53650 4392, Email parkjh07@cu.ac.kr
}

\begin{abstract}
Purpose: The rapidly increasing older adults' population of South Korea has increased the socioeconomic burden on society due to cognitive decline. In this study, we examined the association between changes in physical activity in the older adults and their cognitive function, a primary criterion for the diagnosis of dementia, using panel data from the 2012-2018 Korean Longitudinal Study of Aging.
\end{abstract}

Patients and Methods: This study used baseline (2012) and biennial assessment of follow-up data (2014, 2016, 2018) from Korean Longitudinal Study of Aging. A total of 1733 older adults with the Korean version of the Mini Mental State Examination (K-MMSE) scores in the normal range at the time of the first investigation were included in the study. Physical activity was classified into three categories: inactivity, low physical activity, and high physical activity. Changes in physical activity from 2012 were classified into five groups: remained active, became active in 2016, became active in 2014, became inactive, and remained inactive. Participants' general and health-related characteristics were used as control variables. Descriptive statistics, chi-square tests, and logistic regression analysis were used for analysis.

Results: The baseline mean age of the older adults in 2012 was 71.63 years; of the total 1733 participants, $57.1 \%$ were inactive, $5.7 \%$ had low physical activity, and 37.3\% had high physical activity. Changes in physical activity were significantly associated with cognitive decline. Compared to the group that continued regular physical activity, the risk of cognitive decline increased in the case of participants who became or remained inactive.

Conclusion: Results highlighted the importance of continuous physical activity and its relation to cognitive function. Encouraging continuous physical activity in the older adults has a positive effect on the prevention of cognitive impairment and dementia. Thus, exercise-based interventions should be implemented a community health service.

Keywords: aging, cognition disorders, older adults, physical activity

\section{Introduction}

The number and proportion of older adults are gradually increasing, with the increasing life span of humans due to development of medical technology and the decline in birth rate. ${ }^{1}$ As of 2020, those aged 65 and above comprised $15.7 \%$ of the Korean population. By 2025, South Korea is expected to become a super-aged society, with $20.3 \%$ of the population being over the age of 65 . By 2060, the population of older adults is expected to account for $43.9 \%$ of the total South Korean population. ${ }^{2}$ Cognitive decline and dementia in the older adults are major problems worldwide and are among the primary causes for older people transitioning from communities to medical facilities and hospitals. According to the World Alzheimer Report 2019, ${ }^{3}$ the number of patients with dementia worldwide was estimated to be approximately 50 million in 2017; this number is predicted to be 75 million by 2030, and 131.5 million by 2050. In South Korea, the number of patients with dementia was reported to be approximately 750,000 in 2018, and is estimated to become one million by 2024 and reach two million by $2039 .{ }^{4,5}$ With 12 deaths per 100,000 people, dementia ranks ninth among the major causes of death in South Korea. ${ }^{6}$ Considering the rapid increase in the older adults' population in South Korea, the burden of disease due to dementia is expected to increase in the future. 
The cognitive functions of the older adults change due to genetic factors, age, or the occurrence of diseases, which, in turn, increase the risk of mild cognitive dysfunction or dementia. ${ }^{7}$ For the older adults, cognitive decline is an important problem that seriously affects their health-related quality of life and their overall daily life, to the extent that individuals express a state of loss of purpose in life and fear. ${ }^{8}$ To date, no therapeutic method to reverse and reduce the symptoms of dementia caused by cognitive decline has been found; treatment only alleviates symptoms after the onset of dementia and slows the progression of the disease. ${ }^{9}$ As such, the cognitive decline of the older adults lowers their quality of life, increasing the burden of disease and medical expenses of their families and society.

Appropriate physical activity improves cognitive function, ${ }^{10,11}$ promotes healthy aging, and quality of life, which, in turn, improve quality of sleep and reduce sleep problems. ${ }^{10}$ Particularly, regular physical activity can delay the aging process ${ }^{12}$ lower the mortality, ${ }^{13}$ and enhance the physical and mental health of the older adults. ${ }^{14,15}$ Several studies have examined the relationship between physical activity and cognitive function in the older adults. As per a meta-analysis study, an intervention that combined nutrition with exercise was shown to improve age-related cognitive decline, but not memory, attention and executive function. ${ }^{16}$ Another study on the relationship between sedentary behaviors and cognitive function in the older adults without dementia showed that sedentary behaviors, such as TV viewing, were associated with cognitive decline and had a negative relationship with verbal memory. ${ }^{17}$

In a previous study, the relationship between 5-year prevalence of dementia and physical activity, such as household, leisure, and sport categories, was examined and the findings revealed that a moderate amount of household activity could lower the 5-year prevalence of dementia. ${ }^{18}$ Another study based on cross-sectional data from the Korean Longitudinal Study of Aging (KLoSA) examined the impact of relative grip strength on cognitive function; ${ }^{19}$ a similar research paper attempted to study the impact of physical activity on the health of the older adults. ${ }^{20}$ In the abovementioned crosssectional studies, it was difficult to prove whether changing the level of physical activity was a contributing factor in the cognitive decline of the older adults. Therefore, this study aimed to examine the relationship between changes in physical activity and cognitive function decline with a six-year follow-up after controlling for general and health-related factors in the aged 65 and above. The authors intended to provide evidence that can be used as basic data for prevention and management of cognitive decline in the future, to reduce the national disease burden.

\section{Materials and Methods}

\section{Research Design and Sample}

This research is a secondary retrospective longitudinal panel study examining the changes in physical activity level and cognitive function decline over a six-year period in a nationally representative sample of older South Korean communitydwelling adults. We hypothesized that maintaining or starting physical activity over time is associated with a reduced risk of cognitive function decline, after adjusting the level of physical activity at baseline.

\section{Study Population}

This study used panel survey data from the KLoSA, which is based on the Health and Retirement Survey (HRS) conducted in the USA. ${ }^{21}$ The KLoSA offers longitudinal panel data of South Koreans aged 45 years or older since 2006. This biennial survey uses a stratified and multistage area probability sample of households in South Korea, except Jeju Island, to create a nationally representative sample through computer-assisted personal interviewing. ${ }^{22}$ The KLoSA includes information about a demographic, socioeconomic, and health-related variables. The total sample sizes of the KLoSA were 10,254, 8688, 7486, 7920, 7029, 6618, and 6136 in 2006, 2008, 2010, 2012, 2014, 2016, and 2018, respectively. This study used KLoSA panel data collected from 2012 (fourth wave) to 2018 (seventh wave). We applied the following criteria for sample selection to the total sample size of the KLoSA (7486 participants in 2012): (1) aged 65 years or older, and a Korean version of the Mini Mental State Examination (K-MMSE) score of 24 or higher at baseline, (2) completed the K-MMSE test in 2018, and (3) responded to survey items about physical activity at fourth (2012), fifth (2014), and sixth (2016) waves. Among the 7486 subjects, 5145 were excluded for being below the age of 65 years or having cognitive impairments at baseline. An additional 608 were excluded due to missing data on the MMSE score in 2018 or missing data on physical activity in 2012, 2014, or 2016. The final sample comprised 1733 participants. 


\section{Measurement}

The major outcome measured in this study was changes in cognitive function. The KLoSA used the K-MMSE as a measure of global cognitive function. ${ }^{23}$ The MMSE assesses orientation to place and time; short-term memory; episodic long-term memory; subtraction; the ability to construct a sentence; and oral language ability. ${ }^{24}$ The maximum score was set as 30 , and a score of less than 24 was considered to reflect poor cognition. ${ }^{25}$ The validity of the K-MMSE has been established previously. ${ }^{26}$ In line with the prior study, the present research considered a K-MMSE score of less than 24 in 2018, to reflect a decline in cognitive function.

The KLoSA assessed physical activity by asking whether the subjects participated in any type of exercise at least once a week; if the response was yes, the frequency and duration of exercise were recorded. ${ }^{22}$ Based on the global recommendation, ${ }^{27}$ the level of physical activity was categorized as inactivity, low activity, and high activity. Participants who did not engage in any type of exercise were defined as being inactive; those who exercised 1$149 \mathrm{~min} /$ week were considered to have low activity level; and those who exercised $150 \mathrm{~min} /$ week or over were considered to have high activity level. The independent variable was the change in physical activity over 4 years (2012-2016). Similar to a previous study $^{28}$ that used level of physical activity as a variable, a binary variable (sedentary or low/high activity) was created; we classified physical activity into inactive or active. Previous studies $^{22,29}$ that used the change of physical activity as an independent variable, classified participants into inactive (sedentary), increaser (sedentary to low activity, sedentary to high activity, or low to high activity), decreaser (high to low activity, high activity to sedentary, or low activity to sedentary) to, exerciser (either maintaining high activity or low activity). Based on these previous studies, we categorized a change in physical activity into five groups: "always active," "became active in 2014," "became active in 2016," "became inactive," and "remained inactive." "Remained inactive" referred to respondents who stayed inactive in all three surveys. "Became active in 2014" and "became active in 2016" referred to those who partook in low/high activity from 2014 and 2016 onward, respectively. Subjects who were physically inactive from 2014 or 2016 onward were classified as "became inactive." Finally, subjects who performed low/high activity at the time of the 2012 and 2016 surveys were classified as "remained active."

We selected covariates associated with cognitive function decline among the older adults based on a literature review. All covariates were entered into the analytic model as values at baseline (2012). General characteristics included demographic and socioeconomic factors, such as gender, age, education, having a spouse, living alone, and social participation. Health behavior variables included alcohol consumption, body mass index (BMI), physical activity, and smoking. Instrumental activities of daily living (IADL), comorbidity, and depressive symptoms were also considered as covariates. Age was classified into 65-74 years and 75 years or older. Education level was classified into: elementary school or less, middle school, high school, and college or over. Having a spouse was recoded with a yes or no response; the no classification was further divided into widowed, never married, and separated/divorced. Living alone was identified by response to whether they lived alone or with others. Six types of social activities (eg, alumni societies, church or religious groups, social clubs, sports clubs, volunteer groups, and political parties or non-governmental organizations) were asked in the KLoSA. Subjects answered regarding whether they participated in the activities. Social participation was recorded as $0,1,2$, or more depending on the number of social activities. Smoking was classified into nonsmoker, past smoker, and current smoker; similarly, drinking status was categorized into nondrinkers, past drinkers, and current drinkers. Functional health was assessed using a 10 -item Korean-IADL scale. ${ }^{30}$ Each item was assessed as totally independent, partially dependent, or totally dependent. The IADL was categorized into independent or dependent. If the participant was partially dependent according to even one item, they were classified as dependent. Comorbidity was defined as the sum of the number of physician-diagnosed chronic diseases (hypertension, diabetes mellitus, cancer, chronic lung disease, liver disease, heart disease, cerebrovascular disease, arthritis or rheumatism, psychiatric disease, and prostate disease). Depressive symptoms were assessed using the 10 item Korean version of the Center for Epidemiologic Studies Depression (CESD) scale. The total scores ranged from 0 to 30, with higher scores indicating greater depression. Depressive symptoms were divided into normal and depressive states, based on the cutoff score of ten. 


\section{Data Analysis}

Chi-square tests were performed to determine the baseline level of physical activity and changes in physical activity by covariates. Multiple logistic regression analysis was used to examine the changes in physical activity associated with the MMSE score. In multiple logistic regression analysis, odds ratios (ORs) and 95\% confidence intervals (CIs) were calculated. Analyses were performed using the statistical software SPSS version 26.0 (IBM Corporation, Armonk, USA). All tests were two-tailed, and statistical significance was defined as $p$-value $<0.05$.

\section{Results}

\section{General and Health-Related Characteristics According to the Physical Activity Group}

Table 1 shows participants' results based on the 2012 baseline data. Of the 1733 participants, 878 (50.7\%) were male, and the average age was $71.63 ; 1331(76.8 \%)$ had spouses, and $240(13.8 \%)$ lived alone. Regarding educational background and social activity, $902(52.0 \%)$ graduated elementary school or less, and $1042(60.1 \%)$ participated in at least one social activity. Regarding differences in the general characteristics according to the baseline physical activity-

Table I General Characteristics and Health-Related Characteristics According to the Physical Activity Group at Baseline ( $\mathrm{N}=1733)$

\begin{tabular}{|c|c|c|c|c|c|c|}
\hline Variable & Category & $\begin{array}{l}\text { Total } \\
(n=1733)\end{array}$ & $\begin{array}{l}\text { Inactivity } \\
(n=989)\end{array}$ & $\begin{array}{l}\text { Low Activity } \\
(\mathrm{n}=98)\end{array}$ & $\begin{array}{l}\text { High Activity } \\
(n=646)\end{array}$ & $t / x^{2} / F(p)$ \\
\hline Gender & $\begin{array}{l}\text { Male } \\
\text { Female }\end{array}$ & $\begin{array}{l}878(50.7) \\
855(49.3)\end{array}$ & $\begin{array}{l}438(44.3) \\
551(55.7)\end{array}$ & $\begin{array}{l}46(46.9) \\
52(53.1)\end{array}$ & $\begin{array}{l}394(61.0) \\
252(39.0)\end{array}$ & $44.20(<0.001)$ \\
\hline Age & $\begin{array}{l}M \pm S D \\
65-74 \\
75-\end{array}$ & $\begin{array}{l}71.63 \pm 5 . \mid I \\
1283(74.0) \\
450(26.0)\end{array}$ & $\begin{array}{l}71.8 I \pm 5.14 \\
716(72.4) \\
273(27.6)\end{array}$ & $\begin{array}{l}71.56 \pm 5.35 \\
72(73.5) \\
26(26.5)\end{array}$ & $\begin{array}{l}71.38 \pm 5.02 \\
495(76.6) \\
151(23.4)\end{array}$ & $\begin{array}{l}1.38(0.252) \\
3.65(0.161)\end{array}$ \\
\hline Spouse & $\begin{array}{l}\text { Yes } \\
\text { No }\end{array}$ & $\begin{array}{l}|33|(76.8) \\
402(23.2)\end{array}$ & $\begin{array}{l}734(74.2) \\
255(25.8)\end{array}$ & $\begin{array}{l}75(76.5) \\
23(23.5)\end{array}$ & $\begin{array}{l}522(80.8) \\
124(19.2)\end{array}$ & $9.53(0.009)$ \\
\hline Living arrangement & $\begin{array}{l}\text { Alone } \\
\text { Other }\end{array}$ & $\begin{array}{l}1493(86.2) \\
240(13.8)\end{array}$ & $\begin{array}{l}\text { I5I (I5.3) } \\
838(84.7)\end{array}$ & $\begin{array}{l}12(12.2) \\
86(87.8)\end{array}$ & $\begin{array}{l}77(11.9) \\
569(88.1)\end{array}$ & $3.90(0.143)$ \\
\hline Education level & $\begin{array}{l}\text { University } \\
\text { High school } \\
\text { Middle school } \\
\text { Elementary }\end{array}$ & $\begin{array}{l}170(9.8) \\
365(21.1) \\
296(17.1) \\
902(52.0)\end{array}$ & $\begin{array}{l}55(5.6) \\
15 \mid(15.3) \\
156(15.8) \\
627(63.4)\end{array}$ & $\begin{array}{l}13(13.3) \\
25(25.5) \\
13(13.3) \\
47(48.0)\end{array}$ & $\begin{array}{l}102(15.8) \\
189(29.3) \\
127(19.7) \\
228(35.3)\end{array}$ & $144.19(<0.001)$ \\
\hline Number of social activity participation & $\begin{array}{l}\text { None } \\
1 \\
2 \text { or more }\end{array}$ & $\begin{array}{l}346(20.0) \\
1042(60.1) \\
345(19.9)\end{array}$ & $\begin{array}{l}235(23.8) \\
612(61.9) \\
142(14.4)\end{array}$ & $\begin{array}{l}15(15.3) \\
60(61.2) \\
23(23.5)\end{array}$ & $\begin{array}{l}96(14.9) \\
370(57.3) \\
180(27.9)\end{array}$ & $54.50(<0.00 \mathrm{I})$ \\
\hline Smoking & $\begin{array}{l}\text { Non-smoker } \\
\text { Ex-smoker } \\
\text { Current smoker }\end{array}$ & $\begin{array}{l}1165(67.2) \\
340(19.6) \\
228(13.2)\end{array}$ & $\begin{array}{l}692(70.0) \\
158(16.0) \\
139(14.1)\end{array}$ & $\begin{array}{ll}61 & (62.2) \\
23 & (23.5) \\
14 & (14.3)\end{array}$ & $\begin{array}{l}412(63.8) \\
159(24.6) \\
75(11.6)\end{array}$ & $20.13(<0.001)$ \\
\hline Drinking & $\begin{array}{l}\text { Non-drinker } \\
\text { Ex-drinker } \\
\text { Current drinker }\end{array}$ & $\begin{array}{l}89 \mid(5 I .4) \\
26 I(15.1) \\
58 I(33.5)\end{array}$ & $\begin{array}{l}563(56.9) \\
131(13.2) \\
295(29.8)\end{array}$ & $\begin{array}{l}49(50.0) \\
17(17.3) \\
32(32.7)\end{array}$ & $\begin{array}{l}279(43.2) \\
113(17.5) \\
254(39.3)\end{array}$ & $29.95(<0.001)$ \\
\hline IADL dependency & $\begin{array}{l}\text { Independent } \\
\text { Dependent }\end{array}$ & $\begin{array}{l}1609(92.8) \\
124(7.2)\end{array}$ & $\begin{array}{l}917(92.7) \\
72(7.3)\end{array}$ & $\begin{array}{l}88(89.8) \\
10(10.2)\end{array}$ & $\begin{array}{l}604(93.5) \\
42(6.5)\end{array}$ & I.8I (0.405) \\
\hline K-CESD & $\begin{array}{l}\text { Normal } \\
\text { Depression }\end{array}$ & $\begin{array}{l}884(51.0) \\
849(49.0)\end{array}$ & $\begin{array}{l}456(46.1) \\
533(53.9)\end{array}$ & $\begin{array}{l}53(54.1) \\
45(45.9)\end{array}$ & $\begin{array}{l}375(58.0) \\
27 \mid(42.0)\end{array}$ & $22.69(<0.001)$ \\
\hline Chronic illness & $(\mathrm{M} \pm \mathrm{SD})$ & $1.33 \pm 1.12$ & $1.31 \pm 1.11$ & $1.39 \pm 1.26$ & $1.34 \pm 1.12$ & $0.23(0.79 I)$ \\
\hline BMI & $(\mathrm{M} \pm \mathrm{SD})$ & $23.27 \pm 2.70$ & $23.22 \pm 2.82$ & $23.33 \pm 2.82$ & $23.34 \pm 2.48$ & $0.43(0.653)$ \\
\hline
\end{tabular}


group level, gender $\left(x^{2}=44.20, p<0.001\right)$, having a spouse $\left(x^{2}=10.31, p=0.035\right)$, educational level $\left(x^{2}=144.19\right.$, $p<0.001)$, and the degree of social activity participation $\left(x^{2}=54.50, \mathrm{p}<0.001\right)$ showed significant differences. Regarding health-related characteristics, smoking $\left(x^{2}=20.13, p<0.001\right)$, drinking $\left(x^{2}=29.95, p<0.001\right)$, and depression $\left(x^{2}=22.69, p<0.001\right)$ showed statistically significant differences between the groups with varying physical activity levels.

\section{Change in Level of Physical Activity}

Table 2 shows the differences in the participants' changing levels of physical activity from 2012 to 2018 . Regarding general characteristics, gender $\left(x^{2}=53.86, p<0.001\right)$, age $\left(x^{2}=14.63, p=0.006\right)$, having a spouse $\left(x^{2}=13.74, p=0.008\right)$, educational level $\left(x^{2}=224.85, p<0.001\right)$, and the degree of social activity participation $\left(x^{2}=79.73, p<0.001\right)$ showed a significant difference between the groups, with changes in physical activity. Regarding health-related characteristics, smoking $\left(x^{2}=40.03, p<0.001\right)$, drinking $\left(x^{2}=39.23, p<0.001\right)$, and depression $\left(x^{2}=20.34, p<0.001\right)$ levels were significantly different between the groups which showed changes in physical activity levels.

Table 2 Changes in Physical Activity $(\mathrm{N}=1733)$

\begin{tabular}{|c|c|c|c|c|c|c|c|}
\hline Variable & & $\begin{array}{l}\text { Remained } \\
\text { Active }(n= \\
450)\end{array}$ & $\begin{array}{l}\text { Became } \\
\text { Active at } \\
2016(n=109)\end{array}$ & $\begin{array}{l}\text { Became } \\
\text { Active at } \\
2014(n=114)\end{array}$ & $\begin{array}{l}\text { Became } \\
\text { Inactive ( } \\
=400)\end{array}$ & $\begin{array}{l}\text { Remained } \\
\text { Inactive ( } n \\
=660)\end{array}$ & $x^{2} / F(p)$ \\
\hline Gender & $\begin{array}{l}\text { Male } \\
\text { Female }\end{array}$ & $\begin{array}{l}285(32.5) \\
165(19.3)\end{array}$ & $\begin{array}{l}48(5.5) \\
61(7.1)\end{array}$ & $\begin{array}{l}66(7.5) \\
48(5.6)\end{array}$ & $\begin{array}{l}203(23.1) \\
197(23.0)\end{array}$ & $\begin{array}{l}276(31.4) \\
384(44.9)\end{array}$ & $53.86(<0.001)$ \\
\hline Age & $\begin{array}{l}M \pm S D \\
65-74 \\
75-\end{array}$ & $\begin{array}{l}71.16 \pm 4.77 \\
357(27.8) \\
93(20.7)\end{array}$ & $\begin{array}{l}70.89 \pm 4.82 \\
87(6.8) \\
22(4.9)\end{array}$ & $\begin{array}{l}71.10 \pm 4.64 \\
85(6.6) \\
29(6.4)\end{array}$ & $\begin{array}{l}71.45 \pm 5.32 \\
293(22.8) \\
107(23.8)\end{array}$ & $\begin{array}{l}72.29 \pm 5.27 \\
461(35.9) \\
199(44.2)\end{array}$ & $\begin{array}{l}4.8(0.001) \\
14.63(0.006)\end{array}$ \\
\hline Spouse & $\begin{array}{l}\text { Yes } \\
\text { No }\end{array}$ & $\begin{array}{l}359(27.0) \\
91(22.6)\end{array}$ & $\begin{array}{l}82(6.2) \\
27(6.7)\end{array}$ & $\begin{array}{l}93(7.0) \\
21(5.2)\end{array}$ & $\begin{array}{l}320(24.0) \\
80(19.9)\end{array}$ & $\begin{array}{l}477(35.8) \\
183(45.5)\end{array}$ & I3.74 (0.008) \\
\hline $\begin{array}{l}\text { Living } \\
\text { arrangement }\end{array}$ & $\begin{array}{l}\text { Alone } \\
\text { Other }\end{array}$ & $\begin{array}{l}51(21.3) \\
399(26.7)\end{array}$ & $\begin{array}{l}17(7.1) \\
92(6.2)\end{array}$ & $\begin{array}{l}12(5.0) \\
102(6.8)\end{array}$ & $\begin{array}{l}50(20.8) \\
350(23.4)\end{array}$ & $\begin{array}{l}110(45.8) \\
550(36.8)\end{array}$ & $8.72(0.068)$ \\
\hline Education & $\begin{array}{l}\text { University } \\
\text { High school } \\
\text { Middle school } \\
\text { Elementary }\end{array}$ & $\begin{array}{l}92(54.1) \\
143(39.2) \\
79(26.7) \\
136(15.1)\end{array}$ & $\begin{array}{l}8(4.7) \\
23(6.3) \\
14(4.7) \\
64(7.1)\end{array}$ & $\begin{array}{l}17(10.0) \\
25(6.8) \\
21(7.1) \\
51(5.7)\end{array}$ & $\begin{array}{l}36(21.2) \\
92(25.2) \\
78(26.4) \\
194(21.5)\end{array}$ & $\begin{array}{l}17(10.0) \\
82(22.5) \\
104(35.1) \\
457(50.7)\end{array}$ & $224.85(<0.001)$ \\
\hline $\begin{array}{l}\text { Number of } \\
\text { social activity } \\
\text { participation }\end{array}$ & $\begin{array}{l}\text { None } \\
1 \\
2 \text { or more }\end{array}$ & $\begin{array}{l}50(14.5) \\
259(24.9) \\
14 \mid(40.9)\end{array}$ & $\begin{array}{l}28(8.1) \\
61(5.9) \\
20(5.8)\end{array}$ & $\begin{array}{l}20(5.8) \\
76(7.3) \\
18(5.2)\end{array}$ & $\begin{array}{l}78(22.5) \\
24 I(23 . I) \\
8 I(23.5)\end{array}$ & $\begin{array}{l}170(49.1) \\
409(38.9) \\
85(24.6)\end{array}$ & $79.73(<0.001)$ \\
\hline Smoking & $\begin{array}{l}\text { Non-smoker } \\
\text { Ex-smoker } \\
\text { Current smoker }\end{array}$ & $\begin{array}{l}279(23.9) \\
126(37.1) \\
45(19.7)\end{array}$ & $\begin{array}{l}72(6.2) \\
24(7.1) \\
13(5.7)\end{array}$ & $\begin{array}{l}70(6.0) \\
27(7.9) \\
17(7.5)\end{array}$ & $\begin{array}{l}267(22.9) \\
73(21.5) \\
60(26.3)\end{array}$ & $\begin{array}{l}477(40.9) \\
90(26.5) \\
93(40.8)\end{array}$ & $40.03(<0.001)$ \\
\hline Drinking & $\begin{array}{l}\text { Non-drinker } \\
\text { Ex-drinker } \\
\text { Current drinker }\end{array}$ & $\begin{array}{l}183(20.5) \\
80(30.7) \\
187(32.2)\end{array}$ & $\begin{array}{l}60(6.7) \\
15(5.7) \\
34(5.9)\end{array}$ & $\begin{array}{l}53(5.9) \\
21(8.0) \\
40(6.9)\end{array}$ & $\begin{array}{l}207(23.2) \\
67(25.7) \\
126(21.7)\end{array}$ & $\begin{array}{l}388(43.5) \\
78(29.9) \\
194(33.4)\end{array}$ & $39.23(<0.001)$ \\
\hline $\begin{array}{l}\text { IADL } \\
\text { dependency }\end{array}$ & $\begin{array}{l}\text { Independent } \\
\text { Dependent }\end{array}$ & $\begin{array}{l}426(26.5) \\
24(19.4)\end{array}$ & $\begin{array}{l}102(6.3) \\
7(5.6)\end{array}$ & $\begin{array}{l}106(6.6) \\
8(6.5)\end{array}$ & $\begin{array}{l}366(22.7) \\
34(27.4)\end{array}$ & $\begin{array}{l}609(37.8) \\
5 I(4 I . I)\end{array}$ & $3.75(0.440)$ \\
\hline K-CESD & $\begin{array}{l}\text { Normal } \\
\text { Depression }\end{array}$ & $\begin{array}{l}267(30.2) \\
183(21.6)\end{array}$ & $\begin{array}{l}54(6.1) \\
55(6.5)\end{array}$ & $\begin{array}{l}57(6.4) \\
57(6.7)\end{array}$ & $\begin{array}{l}205(23.2) \\
195(23.0)\end{array}$ & $\begin{array}{l}301(34.0) \\
359(42.3)\end{array}$ & $20.34(<0.001)$ \\
\hline Chronic illness & $(\mathrm{M} \pm \mathrm{SD})$ & $1.32 \pm 1.13$ & $1.17 \pm 1.10$ & $1.47 \pm 1.19$ & $1.35 \pm 1.15$ & $1.32 \pm 1.08$ & $1.06(0.377)$ \\
\hline BMI & $(M \pm S D)$ & $23.25 \pm 2.49$ & $23.39 \pm 2.64$ & $23.42 \pm 2.43$ & $23.57 \pm 2.69$ & $23.05 \pm 2.88$ & $2.40(0.048)$ \\
\hline
\end{tabular}


Table 3 OR $(95 \% \mathrm{Cl})$ for the Association of Physical Activity and Cognitive Decline Over 6 Years Follow-Up $(\mathrm{N}=1733)$

\begin{tabular}{|c|c|c|c|c|}
\hline & Model I OR (95\% CI) & $p$ & Model 2 OR $(95 \% \mathrm{CI})$ & $p$ \\
\hline \multicolumn{5}{|l|}{ Change } \\
\hline Remained active & $\mathrm{I} .00$ (ref) & & 1.00 (ref) & \\
\hline Became active at 2016 & 3.24 (1.67 to 6.29$)$ & 0.001 & 2.56 (1.28 to 5.10$)$ & 0.008 \\
\hline Became active at 2014 & 1.97 (1.00 to 3.88$)$ & 0.051 & I.6I (0.79 to 3.27$)$ & 0.186 \\
\hline Became inactive & 2.60 (1.85 to 3.64$)$ & $<0.001$ & 2.22 (1.56 to 3.17$)$ & $<0.001$ \\
\hline Remained inactive & 2.73 ( 1.56 to 4.77 ) & $<0.001$ & 1.88 (1.04 to 3.38$)$ & 0.035 \\
\hline$-2 \log$ Likelihood & 2043.609 & & 1932.600 & \\
\hline Hosmer-Lemeshow $\chi^{2}$ & 1.603 & 0.979 & 6.375 & 0.605 \\
\hline
\end{tabular}

Notes: Model I: Adjusted by sex, age at baseline. Model 2: Adjusted by sex, age, having spouse, living alone, education, number of chronic disease, BMI, smoking, drinking, depression, IADL, social participation, physical activity at baseline.

\section{Association Between Changes in Physical Activity and Cognitive Decline}

Multiple logistic regression analysis revealed that the regression model was significant; the factors predicting cognitive decline after 6 years of follow-up included: changes in physical activity, old age, low educational level, non-participation in social activities, and depression. The risk of cognitive decline increased by 2.56 times in the case of starting regular physical activity recently, 2.22 times in the case of becoming inactive, and 1.88 times in the case of remaining inactive as compared to the group that had been engaged in regular physical activity. This is while controlling for general characteristics such as gender, age, having a spouse, living alone, educational level, and health-related characteristics such as chronic diseases, BMI, smoking, drinking, depression, IADLs, and social participation (Table 3).

\section{Discussion}

Rising older adults' population and average life expectancy is increasing incidences of dementia. Cognitive decline affects individuals' quality of life, making it impossible for them to live independently, by increasing functional physical activity and decreasing dependence in daily life. This study analyzed the changing associations between physical activity and cognitive function by controlling general and health-related characteristics, and confirmed a significant association between changing levels of physical activity and cognitive function in the older adults.

More than half of the participants' changes in the level of physical activity became or remained inactive. Of the 1733 participants, $25.9 \%$ showed cognitive decline over six years. It can be assumed that other factors, such as an increase in age and chronic diseases, may have influenced this cognitive decline. Hence, we focused on the association between changes in physical activity (ie, remain active since 2012, became active in 2016, became active in 2014, became inactive, remained inactive) with cognitive decline controlling for general and health-related factors. The results showed that maintaining an inactive lifestyle was associated with cognitive decline. However, not all changes in physical activity had a significant effect. After controlling for general and health-related characteristics, those who stopped physical activity had a 2.22 times higher risk of cognitive decline than those who engaged continuously in physical activity; those who did not engage in regular physical activity at all were found to have a 1.88 times higher risk of cognitive decline; this was 2.56 times higher when physical exercise was started in recent 2 years. Our results showed that it is necessary to support the initiation of physical activity as soon as possible and maintain certain period of times, in the case of the older adults who are not currently physically active. Our results, dealing with home-dwelling older adults, also showed the necessity of developing a program that supports continuous physical activity in the community. Accordingly, it is necessary to provide continuous community-based public-funded exercise programs for home-dwelling older adults, ${ }^{31}$ and to uncover the barriers that block their participation in physical activities.

The level of physical activity is a somewhat subjective aspect, since it was measured by participants answering the survey questionnaire. So we considered when the older adults answered the type, frequency, and intensity of physical activity it was considered physically active. However, a previous study emphasized that a moderately physically active lifestyle can contribute to preventing cognitive function decline, while the same benefits were not accrued either from 
light or vigorous physically active lifestyle. ${ }^{32}$ Because regular physical activity functions as a health indicator, the WHO recommends that healthy older adults people over the age of 65 engage, once a week, in at least 2.5 hours of moderate intensity physical activity or one hour and 15 minutes of vigorous aerobic physical activity, unless they have a special medical condition. ${ }^{33}$ Determining whether changes in physical activity for the older adults affect cognitive function, by measuring exercise intensity and time, can reveal the importance of physical activity and the application of a community program that can contribute to a decrease in the risk of cognitive decline. Further studies examining cognitive decline and the degree and category of physical activity, depending on health status, motivation, and leisure status for the older adults, are recommended.

A previous study ${ }^{4}$ investigated the relationship between lifestyle change and cognitive function over 2 years in the South Korean older adults from 2006 to 2016 and lifestyle risk scores were measured with drinking, smoking, excess weight, and exercise participation. This study classified lifestyle change groups as good-good, good-bad, bad-good, and bad-bad groups and showed that the bad-good group had the highest K-MMSE score compared to the bad-bad group; the good-good group showed the second highest result. This study emphasized the importance of maintaining a good lifestyle; it measured cognitive function scores two-year intervals. Therefore, our study is more meaningful in that it studied a continuous dataset for six years, as compared to the previous study.

Considering that the age at which cognitive decline is clearly observed is 65 years and above, and that many studies have been based on this age group, this study selected the subjects aged 65 years or older. The main variables in this study were chosen based on previous studies and comprised general and health-related variables. Each variable was analyzed by categorizing it or defining it as a continuous variable, as described in the variable definition of the research method.

This study has several limitations, in that explaining the causal relationship between changes in physical activity and cognitive decline was difficult, although repeatedly measured data were used as the main variable of interest. It was difficult to ascertain whether changes in physical function precede cognitive decline or, conversely, whether cognitive decline causes changes in physical function. Additionally, due to the limitation of KLoSA data, it was not possible to examine the exact intensity of physical activity. However, despite the limitation of being unable to confirm causality between the two variables, the KLoSA data is a representative sample. With a representative data, this study suggests that changes from baseline physical activity from 2012 to 2018 showed a significant correlation with cognitive function for six years.

Although MMSE has the advantage of being able to quantitatively evaluate cognitive function, it is widely used in various fields and has a high false-negative rate. ${ }^{34}$ Referring to the result that MMSE can mistakenly judge a subject to be in cognitive decline when the level of education is low, ${ }^{34}$ it is suggested that various test tools other than the MMSE score be used comprehensively.

Despite these limitations, this study is meaningful with the following reasons. First, according to the results of KNHANES (Korea National Health and Nutrition Examination Survey), a nationwide, population based, cross-sectional survey regularly conducted by the Korea Centers for Disease Control and Prevention, the rate of regular physical activity in the older adults aged 60 to 69 decreased from $45.7 \%$ in 2015 to $39.3 \%$ in 2019; it showed a slight increase in those aged 70 or over. ${ }^{35}$ Since it is not possible for this cross-sectional survey to accurately represent this trend, our study analyzed the changes in physical activity and any corresponding association with cognitive decline, using longitudinal data. Second, in South Korea, 254 community health centers are installed in each region, and the government recommends the implementation of exercise and physical activity programs for the older adults in these places. ${ }^{36}$ Additionally, the National Health Insurance Service (NHIS), a South Korean single public insurer, is implementing the "Health-100-year Exercise Program" for the older adults to promote health of South Koreans. ${ }^{37}$ Our study examined the implications such that these publicly funded programs have practical effects.

\section{Conclusion}

This study examined the effect of changes in physical activity on cognitive decline in South Korean older adults. Six years of data from the KLoSA, consisting of the elderly aged 65 years or older without any evidence of cognitive decline as of 2012, were analyzed. The study showed that the risk of cognitive decline increased with an inactive lifestyle and confirmed a significant association between changes in physical activity and cognitive function decline in older adults. 
Further studies are needed to find the point at which becoming active can prevent cognitive decline. Based on the results, it is necessary to emphasize the importance of continuous exercise for the older adults and encourage exercise. To that end, the support of the community is crucial.

\section{Abbreviations}

BMI, body mass index; CESD, center for epidemiologic studies depression scale; CI, confidence interval; IADL, instrumental activities of daily living; K-MMSE, Korean mini mental state examination; KLoSA, Korean longitudinal study of aging; OR, odds ratio; WHO, World Health Organization.

\section{Data Sharing Statement}

Data available on request due to ethical considerations; the data presented in this research are available on request from the corresponding author.

\section{Ethics Approval and Informed Consent}

This study was approved by Daegu Catholic University Institutional Review Board (approval number: CUIRB-2020E001). This study was a secondary data analysis study that was open to the public so it was exempt from informed consent.

\section{Author Contributions}

All authors made a significant contribution to the work reported, whether that is in the conception, study design, execution, acquisition of data, analysis and interpretation, or in all these areas; took part in drafting, revising or critically reviewing the article; gave final approval of the version to be published; have agreed on the journal to which the article has been submitted; and agree to be accountable for all aspects of the work.

\section{Funding}

This research received no specific grant from any funding agency in the public, commercial, or not-for-profit sectors.

\section{Disclosure}

The authors declare no competing interest.

\section{References}

1. Global Health and Aging. National Institute on Aging, National Institutes of Health, U.S. Department of Health and Human Services, and World Health Organization. Available from: https://www.who.int/ageing/publications/global_health.pdf. Accessed September 2, 2021.

2. Korean Statistical Information Service. Senior statistics; 2020. Available from: http://kostat.go.kr/portal/korea/kor_nw/1/1/index.board?bmode= read\&aSeq=385322. Accessed June 11, 2021.

3. Alzheimer's Disease International. World Alzheimer report 2019: attitudes to dementia. Available from: https://www.alzint.org/u/ WorldAlzheimerReport2019.pdf. Accessed May 18, 2021.

4. Lee HJ, Jang J, Choi DW, Chae W, Park EC, Jang SI. Association between change in lifestyle and cognitive functions among elderly Koreans: findings from the Korean longitudinal study of aging (2006-2016). BMC Geriatr. 2020;20(1):317. doi:10.1186/s12877-020-01693-7

5. Lee J, Kang M, Nam H, Kim Y, Lee O, Kim K Korean Dementia Observatory 2019 (NIDR-1902-0028). Department of Health, National Institute of Dementia; 2020. Available from: https://www.nid.or.kr/info/dataroom_view.aspx?bid=209. Accessed June 11, 2021.

6. Statistics Korea. Statistics on causes of death in 2018; 2019. Available from: http://kostat.go.kr/portal/korea/kor_nw/1/6/2/index.board?bmode= read\&bSeq=\&aSeq=377606\&pageNo=1\&rowNum=10\&navCount=10\&currPg=\&searchInfo=\&sTarget=title\&sTxt=. Accessed June 11, 2021.

7. Ferrari C, Lombardi G, Polito C, et al. Alzheimer's disease progression: factors influencing cognitive decline. J Alzheimers Dis. 2018;61 (2):785-791. doi:10.3233/JAD-170665

8. Eisenhauer CM, Pullen CH, Hunter JL, Nelson T. The influence of cognitive decline on rural identity: perspectives of older women. J Holist Nurs. 2015;33(2):134-145. doi:10.1177/0898010114544218

9. Kane RL, Ouslander JG, Resnick B, Malone ML. Essentials of Clinical Geriatrics. 8th ed. New York: McGraw-Hill Education; 2018.

10. Bademli K, Lok N, Canbaz M, Lok S. Effects of physical activity program on cognitive function and sleep quality in elderly with mild cognitive impairment: a randomized controlled trial. Perspect Psychiatr Care. 2019;55(3):401-408. doi:10.1111/ppc.12324

11. Suzuki K, Niimura H, Kida H, et al. Increasing light physical activity helps to maintain cognitive function among the community-dwelling oldest old population: a cross-sectional study using actigraph from the Arakawa 85+ study. Geriatr Gerontol Int. 2020;20(8):773-778. doi:10.1111/ ggi.13967 
12. Kwon G. The role and expectations of physical education for the elderly for active aging in the age of 100 . Korean $J$ Growth Dev. $2020 ; 28$ (3):381-388. doi:10.34284/KJGD.2020.08.28.3.381

13. Oida Y, Kitabatake Y, Nishijima Y, et al. Effects of a 5-year exercise-centered health-promoting programme on mortality and ADL impairment in the elderly. Age Ageing. 2003;32(6):585-592. doi:10.1093/ageing/afg123

14. Coudert J, Van Praagh E. Endurance exercise training in the elderly: effects on cardiovascular function. Curr Opin Clin Nutr Metab Care. 2000;3 (6):479-483. doi:10.1097/00075197-200011000-00010

15. Jia RX, Liang JH, Xu Y, Wang YQ. Effects of physical activity and exercise on the cognitive function of patients with Alzheimer disease: a meta-analysis. BMC Geriatr. 2019;19(1):181. doi:10.1186/s12877-019-1175-2

16. Liu T, Li N, Hou Z, et al. Nutrition and exercise interventions could ameliorate age-related cognitive decline: a meta-analysis of randomized controlled trials. Aging Clin Exp Res. 2021;33:1799-1809. doi:10.1007/s40520-020-01730-w

17. Fancourt D, Steptoe A. Television viewing and cognitive decline in older age: findings from the English Longitudinal Study of Ageing. Sci Rep. 2019;9(1):2851. doi:10.1038/s41598-019-39354-4

18. Dupre C, Bongue B, Helmer C, et al. Physical activity types and risk of dementia in community-dwelling older people: the three-City cohort. BMC Geriatr. 2020;20(1):132. doi:10.1186/s12877-020-01538-3

19. Choi M, Kim G. The effects of relative handgrip strength on cognitive function: moderating roles of paid employment status and social activities. Hanguk Nonyonhak. 2019;39(3):549-567. doi:10.31888/JKGS.2019.39.3.549

20. Oh S, Cho I. A study on the effect of physical exercise on health. Korean Data Anal Soc. 2018;20(3):1381-1394. doi:10.37727/ jkdas.2018.20.3.1381

21. Choi H, Kim K, Park J, Lee S. Analysis Report of KLoSA. Seoul: Korea Employment Information Service; 2019.

22. Lee Y, Kim J, Han ES, et al. Changes in physical activity and cognitive decline in older adults living in the community. Age (Dordr). 2015;37(2):20. doi:10.1007/s11357-015-9759-z

23. Min JW. A longitudinal study of cognitive trajectories and its factors for Koreans aged 60 and over: a latent growth mixture model. Int $J$ Geriatr Psychiatry. 2018;33(5):755-762. doi:10.1002/gps.4855

24. Folstein MF, Folstein SE, McHugh PR. "Mini-mental state". A practical method for grading the cognitive state of patients for the clinician. J Psychiatr Res. 1975;12(3):189-198. doi:10.1016/0022-3956(75)90026-6

25. Anthony JC, LeResche L, Niaz U, von Korff MR, Folstein MF. Limits of the 'Mini-Mental State' as a screening test for dementia and delirium among hospital patients. Psychol Med. 1982;12(2):397-408. doi:10.1017/s0033291700046730

26. Kang Y, Na DL, Hahn S. A validity study on the Korean Mini-Mental State Examination (K-MMSE) in dementia patients. J Korean Neurol Assoc. 1997;15(2):300-308.

27. World Health Organization. Global Recommendations on Physical Health Activity for Health. Geneva: World Health Organization; 2010.

28. Hamer M, Lavoie KL, Bacon SL. Taking up physical activity in later life and healthy ageing: the English longitudinal study of ageing. Br J Sports Med. 2014;48(3):239-243. doi:10.1136/bjsports-2013-092993

29. Berk DR, Hubert HB, Fries JF. Associations of changes in exercise level with subsequent disability among seniors: a 16-year longitudinal study. J Gerontol A Biol Sci Med Sci. 2006;61(1):97-102. doi:10.1093/gerona/61.1.97

30. Won CW, Rho YG, Duk S, Lee YS. The validity and reliability of Korean Instrumental Activities of Daily Living (K-IADL) scale. $J$ Korean Geriatr Soc. 2002;6(4):273-280.

31. Lee S, Park Won Y, Park H. Improvement plan of 'Health-100-year Exercise Program' for the senior citizen. Korean Soc Sociol Sport. 2018;31 (1): $1-23$.

32. Law LL, Rol RN, Schultz SA, et al. Moderate intensity physical activity associates with CSF biomarkers in a cohort at risk for Alzheimer's disease. Alzheimers Dement (Amst). 2018;10:188-195. doi:10.1016/j.dadm.2018.01.001

33. World Health Organization. Physical activity; 2020. Available from: https://www.who.int/news-room/fact-sheets/detail/physical-activity. Accessed June 10, 2021.

34. Ranson JM, KuKuo E, Hamilton W, Muniz-Terrera G, Langa KM, Llewellyn DJ. Predictors of dementia misclassification when using brief cognitive assessments. Neurol Clin Pract. 2019;9(2):109-117. doi:10.1212/CPJ.0000000000000566

35. Ministry of Health, Korean Disease Control and Prevention Agency. 2019 Korean Health Statistics. Seojong: KDCA; 2020. Available from: https:// knhanes.kdca.go.kr/knhanes/eng/index.do. Accessed June 11, 2021.

36. Han S, Lee Y, Cho C, Im M. A systematic review of literature on community health center exercise program. J Korean Acad Community Health Nurs. 2015;26(1):18-30. doi:10.12799/jkachn.2015.26.1.18

37. Lee JY, Lee HS, Koh KW, Son KJ. Improvement direction of health promotion program of Korea National Insurance Service. Korean J Health Educ Promot. 2018;35(2):123-131. doi:10.14367/kjhep.2018.35.2.123

Journal of Multidisciplinary Healthcare

Dovepress

\section{Publish your work in this journal}

The Journal of Multidisciplinary Healthcare is an international, peer-reviewed open-access journal that aims to represent and publish research in healthcare areas delivered by practitioners of different disciplines. This includes studies and reviews conducted by multidisciplinary teams as well as research which evaluates the results or conduct of such teams or healthcare processes in general. The journal covers a very wide range of areas and welcomes submissions from practitioners at all levels, from all over the world. The manuscript management system is completely online and includes a very quick and fair peer-review system. Visit http://www.dovepress.com/testimonials.php to read real quotes from published authors.

Submit your manuscript here: https://www.dovepress.com/journal-of-inflammation-research-journal

Journal of Multidisciplinary Healthcare 2022:15

$f y$ in $>$ DovePress 